\title{
Heavy MSSM Higgs Interpretation of the LHC Run I Data
}

\section{S. Heinemeyer*}

Campus of International Excellence UAM+CSIC, Cantoblanco, Madrid, Spain; Instituto de Física Teórica UAM-CSIC, C/ Nicolas Cabrera 13-15, Madrid, Spain; Instituto de Física de Cantabria (CSIC-UC), Santander, Spain

E-mail: Sven.Heinemeyer@cern.ch

We review that the heavy $\mathscr{C} \mathscr{P}$-even MSSM Higgs boson is still a viable candidate to explain the Higgs signal at $125 \mathrm{GeV}$. This is possible in a highly constrained parameter region, that will be probed by LHC searches for the $\mathscr{C} \mathscr{P}$-odd Higgs boson and the charged Higgs boson in the near future. We briefly discuss the new benchmark scenarios that can be employed to maximize the sensitivity of the experimental analysis to this interpretation.

Prospects for Charged Higgs Discovery at Colliders

3-6 October 2016

Uppsala University, Sweden

${ }^{*}$ Speaker. 


\section{Introduction}

The discovery of a SM-like Higgs boson in Run I of the Large Hadron Collider (LHC) $[1,2]$ marks a milestone in the exploration of electroweak symmetry breaking (EWSB). Within experimental and theoretical uncertainties, the properties of the new particle are compatible with the Higgs boson of the Standard Model (SM) [3]. Looking beyond the SM, also the light $\mathscr{C} \mathscr{P}$-even Higgs boson of the Minimal Supersymmetric Standard Model (MSSM) [4] is a perfect candidate, as it possesses SM Higgs-like properties over a significant part of the model parameter space with only small deviations from the SM in the Higgs production and decay rates [5].

Here we will review [6] that also the heavy $\mathscr{C} \mathscr{P}$-even Higgs boson of the MSSM is a viable candidate to explain the observed signal at $125 \mathrm{GeV}$. (the "heavy Higgs case", which has been discussed in Refs. [5-11]). At lowest order, the Higgs sector of the MSSM can be fully specified in terms of the $W$ and $Z$ boson masses, $M_{W}$ and $M_{Z}$, the $\mathscr{C} \mathscr{P}$-odd Higgs boson mass, $M_{A}$, and $\tan \beta \equiv v_{2} / v_{1}$, the ratio of the two neutral Higgs vacuum expectation values. However, higherorder corrections are crucial for a precise prediction of the MSSM Higgs boson properties and introduce dependences on other model parameters, see e.g. Refs. [12-14] for reviews.

In the heavy Higgs case all five MSSM Higgs bosons are relatively light, and in particular the lightest $\mathscr{C} \mathscr{P}$-even Higgs boson has a mass (substantially) smaller than $125 \mathrm{GeV}$ with suppressed couplings to gauge bosons. We review whether the heavy Higgs case in the MSSM can still provide a good theoretical description of the current experimental data, and which parts of the parameter space of the MSSM are favored. We also discuss the newly defined benchmark scenarios in which this possibility is realized, in agreement with all current Higgs constraints.

\section{Theoretical basis}

In the supersymmetric extension of the SM, an even number of Higgs multiplets consisting of pairs of Higgs doublets with opposite hypercharge is required to avoid anomalies due to the supersymmetric Higgsino partners. Consequently the MSSM employs two Higgs doublets, denoted by $H_{1}$ and $H_{2}$, with hypercharges -1 and +1 , respectively. After minimizing the scalar potential, the neutral components of $H_{1}$ and $H_{2}$ acquire vacuum expectation values (vevs), $v_{1}$ and $v_{2}$. Without loss of generality, one can assume that the vevs are real and non-negative, yielding

$$
v^{2} \equiv v_{1}^{2}+v_{2}^{2} \simeq(246 \mathrm{GeV})^{2}, \quad \tan \beta \equiv v_{2} / v_{1} .
$$

The two-doublet Higgs sector gives rise to five physical Higgs states. Neglecting $\mathscr{C} \mathscr{P}$-violating phases the mass eigenstates correspond to the neutral $\mathscr{C} \mathscr{P}$-even Higgs bosons $h, H$ (with $M_{h}<$ $M_{H}$ ), the $\mathscr{C} \mathscr{P}$-odd $A$, and the charged Higgs pair $H^{ \pm}$.

At lowest order, the MSSM Higgs sector is fully described by $M_{Z}$ and two MSSM parameters, conveniently chosen as $M_{A}$, and $\tan \beta$. Higher order corrections to the Higgs masses are known to be sizable and must be included, in order to be consistent with the observed Higgs signal at $125 \mathrm{GeV}$ [3]. In order to shift the mass of $h$ up to $125 \mathrm{GeV}$, large radiative corrections are necessary, which require a large splitting in the stop sector and/or heavy stops. The stop (sbottom) sector is governed by the soft SUSY-breaking mass parameter $M_{\tilde{t}_{L}}$ and $M_{\tilde{t}_{R}}\left(M_{\tilde{b}_{L}}\right.$ and $\left.M_{\tilde{b}_{R}}\right)$, where 
SU(2) gauge invariance requires $M_{\tilde{t}_{L}}=M_{\tilde{b}_{L}}$, the trilinear coupling $A_{t}\left(A_{b}\right)$ and the Higgsino mass parameter $\mu$.

The "heavy Higgs case", i.e. the heavy $\mathscr{C} \mathscr{P}$-even Higgs boson gives rise to the signal observed at $125 \mathrm{GeV}$ can only be realized in the alignment without decoupling limit. In the so-called Higgs basis (see Ref. [6] for details and citations), the scalar Higgs potential in terms of the Higgs basis fields $\mathscr{H}_{1}$ and $\mathscr{H}_{2}$, can be expressed as

$$
\mathscr{V}=\ldots+\frac{1}{2} Z_{1}\left(\mathscr{H}_{1}^{\dagger} \mathscr{H}_{1}\right)^{2}+\ldots+\left[Z_{5}\left(\mathscr{H}_{1}^{\dagger} \mathscr{H}_{2}\right)^{2}+Z_{6}\left(\mathscr{H}_{1}^{\dagger} \mathscr{H}_{1}\right)\left(\mathscr{H}_{1}^{\dagger} \mathscr{H}_{2}\right)+\text { h.c. }\right]+\ldots
$$

where the most important terms of the scalar potential are highlighted above. The quartic couplings $Z_{1}, Z_{5}$ and $Z_{6}$ are linear combinations of the quartic couplings that appear in the MSSM Higgs potential expressed in terms of $H_{1}$ and $H_{2}$. The $Z_{i}$ are $\mathscr{O}(1)$ parameters.

The mass matrix of the neutral $\mathscr{C} \mathscr{P}$-even Higgs bosons is then given by

$$
\mathscr{M}^{2}=\left(\begin{array}{cc}
Z_{1} v^{2} & Z_{6} v^{2} \\
Z_{6} v^{2} & M_{A}^{2}+Z_{5} v^{2}
\end{array}\right) .
$$

The alignment without decoupling limit is reached for $\left|Z_{6}\right| \ll 1$. In this case $h$ is SM-like if $M_{A}^{2}+$ $\left(Z_{5}-Z_{1}\right) v^{2}>0$ and $H$ is SM-like if $M_{A}^{2}+\left(Z_{5}-Z_{1}\right) v^{2}<0$ : the "heavy Higgs case".

The possibility of alignment without decoupling has been analyzed in detail in Refs. [15-22] (see also the " $\tau$-phobic" benchmark scenario in Ref. [23]). It was pointed out that exact alignment via $\left|Z_{6}\right| \ll 1$ can only happen through an accidental cancellation of the tree-level terms with contributions arising at the one-loop level (or higher).

\section{Parameter scan and observables}

The results shown below have been obtained by scanning the MSSM parameter space. To achieve a good sampling of the full MSSM parameter space with $\mathscr{O}\left(10^{7}\right)$ points, we restrict ourselves to the eight MSSM parameters, called the pMSSM 8,

$$
\tan \beta, \quad M_{A}, \quad M_{\tilde{q}_{3}}, \quad A_{f}, \quad \mu, \quad M_{\tilde{\ell}_{3}}, \quad M_{\tilde{\ell}_{1,2}}, \quad M_{2},
$$

most relevant for the phenomenology of the Higgs sector. Here $\mu$ denotes the Higgs mixing parameter, $M_{\tilde{\ell}_{3}}\left(M_{\tilde{\ell}_{1,2}}\right)$ is the diagonal soft SUSY-breaking parameters for scalar leptons in the thrid (second and first) generation, and $M_{2}$ denotes the SU(2) gaugino soft SUSY-breaking parameter. The scan assumes furthermore that the third generation squark and slepton parameters are universal. That is, we take $M_{\tilde{q}_{3}}:=M_{\tilde{t}_{L}}\left(=M_{\tilde{b}_{L}}\right)=M_{\tilde{t}_{R}}=M_{\tilde{b}_{R}}, M_{\tilde{\ell}_{3}}:=M_{\tilde{\tau}_{L}}=M_{\tilde{\tau}_{R}}=M_{\tilde{v}_{\tau}}$ and $A_{f}:=A_{t}=A_{b}=A_{\tau}$. The remaining MSSM parameters are fixed,

$$
\begin{array}{r}
M_{\tilde{q}_{L}}=M_{\tilde{q}_{R}}(q=c, s, u, d)=1500 \mathrm{GeV}, \\
M_{3}=m_{\tilde{g}}=1500 \mathrm{GeV} .
\end{array}
$$

The high values for the squark and gluino mass parameters, which have a minor impact on the Higgs sector, are chosen in order to be in agreement with the limits from direct SUSY searches. The U(1) gaugino mass parameter is fixed via the usual GUT relation. The pMSSM 8 parameter 


\begin{tabular}{|r|cc|}
\hline Parameter & Minimum & Maximum \\
\hline$M_{A}[\mathrm{GeV}]$ & 90 & 200 \\
$\tan \beta$ & 1 & 20 \\
$M_{\tilde{q}_{3}}[\mathrm{GeV}]$ & 200 & 1500 \\
$M_{\tilde{\ell}_{3}}[\mathrm{GeV}]$ & 200 & 1000 \\
$M_{\tilde{\ell}_{1,2}}[\mathrm{GeV}]$ & 200 & 1000 \\
$\mu[\mathrm{GeV}]$ & -5000 & 5000 \\
$A_{f}[\mathrm{GeV}]$ & $-3 M_{\tilde{q}_{3}}$ & $3 M_{\tilde{q}_{3}}$ \\
$M_{2}[\mathrm{GeV}]$ & 200 & 500 \\
\hline
\end{tabular}

Table 1: Ranges used for the free parameters in the pMSSM 8 scan.

space is scanned with uniformly distributed random values in the eight input parameters over the parameter ranges given in Tab. 1.

We calculate the SUSY particle spectrum and the MSSM Higgs masses using FeynHiggs (version 2.11.2) ${ }^{1}$ [24-27], and estimate the remaining theoretical uncertainty (e.g. from unknown higher-order corrections) in the Higgs mass calculation to be $3 \mathrm{GeV}$ [26]. Following Refs. [8, 11], we demand that all points fulfill a $\mathbf{Z}$-matrix criterion, ||$Z_{21}^{2 \mathrm{~L}}|-| Z_{21}^{1 \mathrm{~L}}|| /\left|Z_{21}^{1 \mathrm{~L}}\right|<0.25$ in order to ensure a reliable and stable perturbative behavior in the calculation of propagator-type contributions in the MSSM Higgs sector. The $\mathbf{Z}$-matrix definition and details can be found in Ref. [27].

The observables included in the fit are the Higgs-boson mass, the Higgs signal rates (evaluated with HiggsSignals [28]), Higgs exclusion bounds from LEP, Tevatron and the LHC (evaluated with HiggsBounds [29]), SUSY exclusion bounds from the LEP and the LHC (the latter evaluated with CheckMate [30]), and several low-energy observables (LEOs): $\operatorname{BR}\left(B \rightarrow X_{s} \gamma\right)$, $\operatorname{BR}\left(B_{s} \rightarrow \mu^{+} \mu^{-}\right)$and $\operatorname{BR}\left(B_{u} \rightarrow \tau \nu_{\tau}\right)$ (evaluated with SuperIso [31]), $(g-2)_{\mu}$ (evaluated with SuperIso and FeynHiggs), and $M_{W}$ (with an evaluation based on Ref. [32]). The total $\chi^{2}$ is evaluated as (see Ref. [6] for more details),

$$
\chi_{H}^{2}=\frac{\left(M_{H}-\hat{M}_{H}\right)^{2}}{\sigma_{\hat{M}_{H}}^{2}}+\chi_{\mathrm{HS}}^{2}+\sum_{i=1}^{n_{\mathrm{LEO}}} \frac{\left(O_{i}-\hat{O}_{i}\right)^{2}}{\sigma_{i}^{2}}-2 \ln \mathscr{L}_{\text {limits }},
$$

where experimental measurements are denoted with a hat.

\section{Results for the "heavy Higgs case"}

Based on the above described $\chi^{2}$ evaluation the best-fit point, shown as a star below, and the preferred parameter regions are derived. Points with $\Delta \chi_{H}^{2}<2.30(5.99)$ are highlighted in red (yellow), corresponding to points in a two-dimensional 68\% (95\%) C.L. region in the Gaussian limit. The best fit point has a $\chi^{2} /$ dof of $73.7 / 85$, corresponding to a $p$-value of 0.87 , i.e. the heavy Higgs case presents an excellent fit to the experimental data [6].

\footnotetext{
${ }^{1}$ Recent updates in the Higgs boson mass calculations [24] lead to a downward shift in $M_{h}$, in particular for large values of $X_{t} / M_{S}$. These changes range within the estimated uncertainties and should not have a drastic impact on our analysis.
} 
In Fig. 1 [6] we review the correlations for the heavy Higgs signal rates,

$$
R_{X X}^{P(H)}=\frac{\sum_{P(H)} \sigma(P(H)) \times \mathrm{BR}(H \rightarrow X X)}{\sum_{P(H)} \sigma_{\mathrm{SM}}(P(H)) \times \mathrm{BR}_{\mathrm{SM}}(H \rightarrow X X)} .
$$

Here $X X=V V, \gamma \gamma, b b, \tau \tau$ (with $V=W^{ \pm}, Z$ ) denotes the final state from the Higgs decay and $P(H)$ denotes the Higgs production mode. It can be seen that the heavy Higgs case can reproduce the SM case $\left(R_{X X}^{P(H)}=1\right)$, but also allows for some spread, in particular in $R_{\tau \tau}^{H}$.
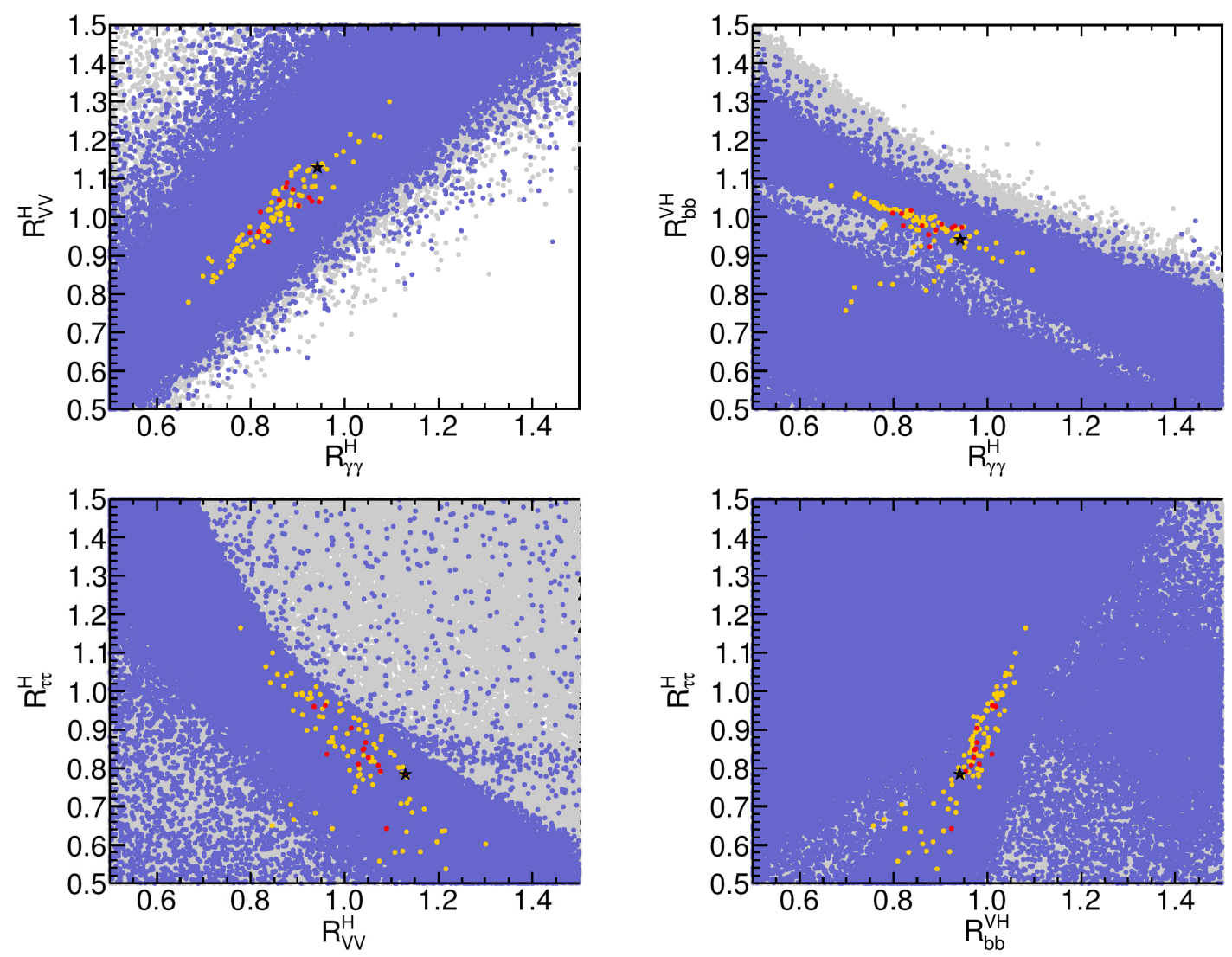

Figure 1: Correlations between signal rates for the heavy Higgs case. The best-fit points are shown as a black star, and points with $\Delta \chi_{H}^{2}<2.3$ (shown in red) and $\Delta \chi_{H}^{2}<5.99$ (shown in yellow).

The MSSM parameter space for the heavy Higgs scenario is shown in Fig. 2. The left plot indicates the preferred regions in the $M_{A}$-tan $\beta$ plane, where one can see that $140 \mathrm{GeV} \lesssim M_{A} \lesssim 185 \mathrm{GeV}$ must be fulfilled, while $\tan \beta$ ranges between $\sim 6$ and $\sim 11$. The right plot shows the preferred regions in the $X_{t} / M_{S}-m_{\tilde{t}_{1}}$ plane. Here the heavy Higgs case makes a clear prediction with $300 \mathrm{GeV} \lesssim$ $m_{\tilde{t}_{1}} \lesssim 650 \mathrm{GeV}$ and $X_{t} / M_{S} \sim-1.5$. Some properties of the light $\mathscr{C} \mathscr{P}$-even Higgs boson are shown in Fig. 3. The left plot shows the light Higgs boson coupling to massive gauge bosons relative to the SM value. One can see that the coupling squared is suppressed by a factor of 1000 or more, rendering its discovery via $e^{+} e^{-} \rightarrow Z^{*} \rightarrow Z h$ at LEP impossible [33,34]. The right plot gives the $\mathrm{BR}(H \rightarrow h h)$ for $M_{h} \lesssim M_{H} / 2$. Here it is shown that the BR does not exceed 20\%, and thus does not distort the coupling measurements of the heavy Higgs at $\sim 125 \mathrm{GeV}$ too much [3]. 

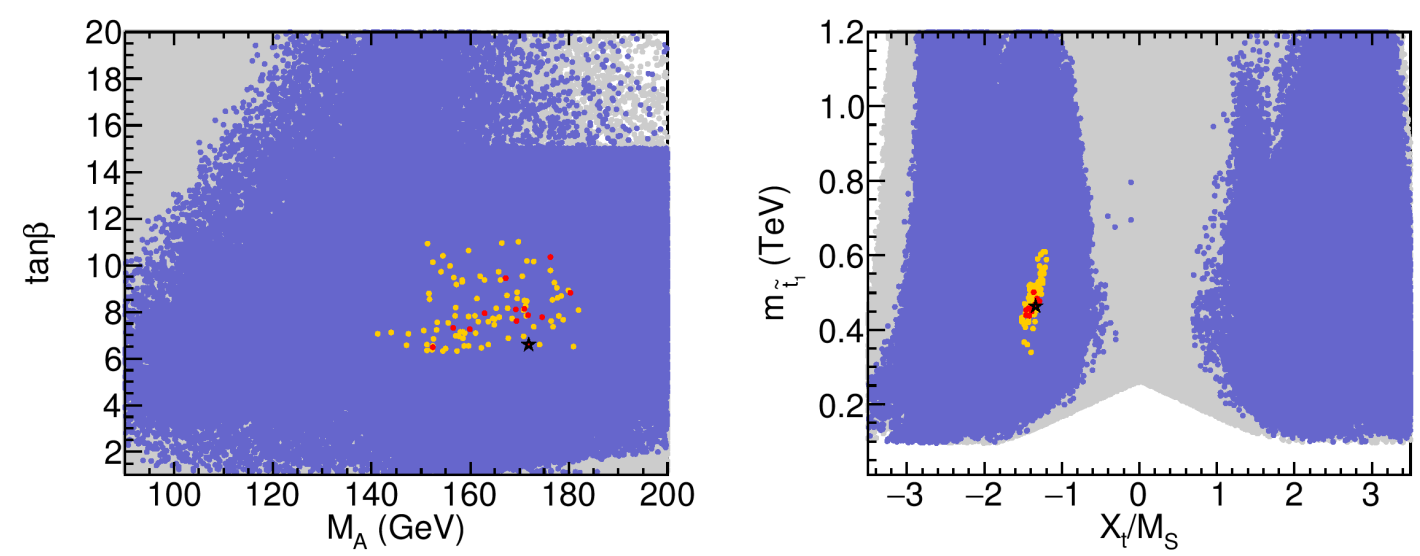

Figure 2: $M_{A}-\tan \beta$ plane (left) and $X_{t} / M_{S}-m_{\tilde{t}_{1}}$ plane (right) in the heavy Higgs case. The color coding is as in Fig. 1.
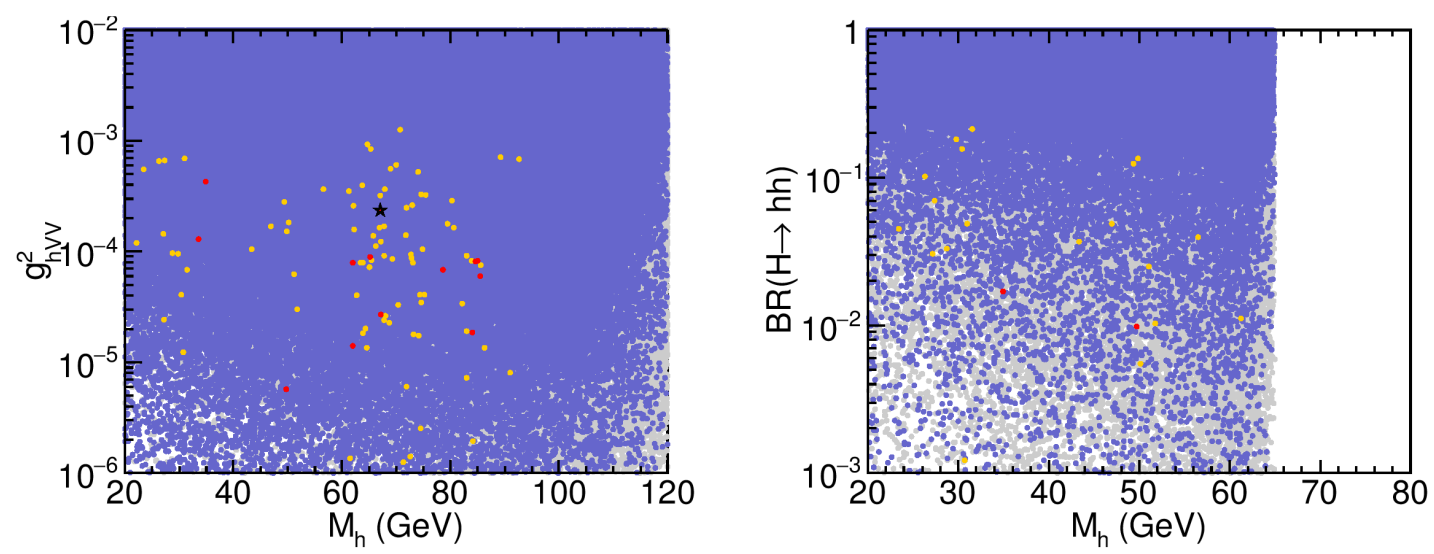

Figure 3: $g_{h V V}^{2}$ (relative to the $\mathrm{SM}$ value) (left) and $\mathrm{BR}(H \rightarrow h h)$ as a function of $M_{h}$ (right) in the heavy Higgs case. The color coding is as in Fig. 1.

\section{Updated benchmark scenarios}

In Ref. [6] an updated set of benchmarks for the heavy Higgs case was presented, superseeding the experimentally excluded low- $M_{H}$ scenario [23]. The parameters of the three new benchmark scenarios are given in Tab. 2 . The low- $M_{H}^{\text {alt- }}\left(\right.$ low- $\left.M_{H}^{\text {alt }}\right)$ ) scenario is defined in the $\mu$-tan $\beta$ plane with $M_{H^{ \pm}}<(>) m_{t}$, while the low- $M_{H}^{\text {altv }}$ scenario has a fixed $\mu$ in the $M_{H^{ \pm}}-\tan \beta$ plane.

The experimentally allowed parameter space in the three benchmark scenarios is shown in Fig. $4{ }^{2}$ The red, orange and blue regions are disfavoured at the 95\% C.L. by LEP light Higgs $h$ searches [34], LHC $H / A \rightarrow \tau^{+} \tau^{-}$searches [35,36] and LHC $t \rightarrow H^{+} b \rightarrow(\tau \nu) b$ searches [37,38], respectively. The green area indicates parameter regions that are compatible with the Higgs signal (at $\sim 95 \%$ C.L., see Ref. [6] for details), unphysical regions are displayed in gray. Contour lines indicate the Higgs masses $M_{h}$ and $M_{H}$ (in GeV).

\footnotetext{
${ }^{2}$ In the evaluation of these plots the two-loop corrections to the $M_{A}-M_{H^{ \pm}}$mass relation had been omitted. Taking them into account will lead to a slight shift of the $M_{h}$ contour lines.
} 


\begin{tabular}{lccc}
\hline Benchmark scenario & $M_{H^{ \pm}}[\mathrm{GeV}]$ & $\mu[\mathrm{GeV}]$ & $\tan \beta$ \\
\hline low- $M_{H}^{\text {alt- }}$ & 155 & $3800-6500$ & $4-9$ \\
low- $M_{H}^{\text {alt }}$ & 185 & $4800-7000$ & $4-9$ \\
low- $M_{H}^{\text {altv }}$ & $140-220$ & 6000 & $4-9$ \\
\hline fixed parameters: & $m_{t}=173.2 \mathrm{GeV}, \quad A_{t}=A_{\tau}=A_{b}=-70 \mathrm{GeV}, \quad M_{2}=300 \mathrm{GeV}$, \\
& $M_{\tilde{q}_{L}}=M_{\tilde{q}_{R}}=1500 \mathrm{GeV}(q=c, s, u, d), \quad m_{\tilde{g}}=1500 \mathrm{GeV}$, \\
& $M_{\tilde{q}_{3}}=750 \mathrm{GeV}, \quad M_{\tilde{\ell}_{1,2}}=250 \mathrm{GeV}, \quad M_{\tilde{\ell}_{3}}=500 \mathrm{GeV}$ \\
\hline
\end{tabular}

Table 2: Parameters of the updated low- $M_{H}$ benchmark scenarios, see Ref. [6] for more details. The lower row gives the fixed parameters that are common to all three benchmark scenarios and $M_{1}=\frac{5}{3} \frac{s_{\mathrm{w}}^{2}}{c_{\mathrm{W}}^{2}} M_{2}$
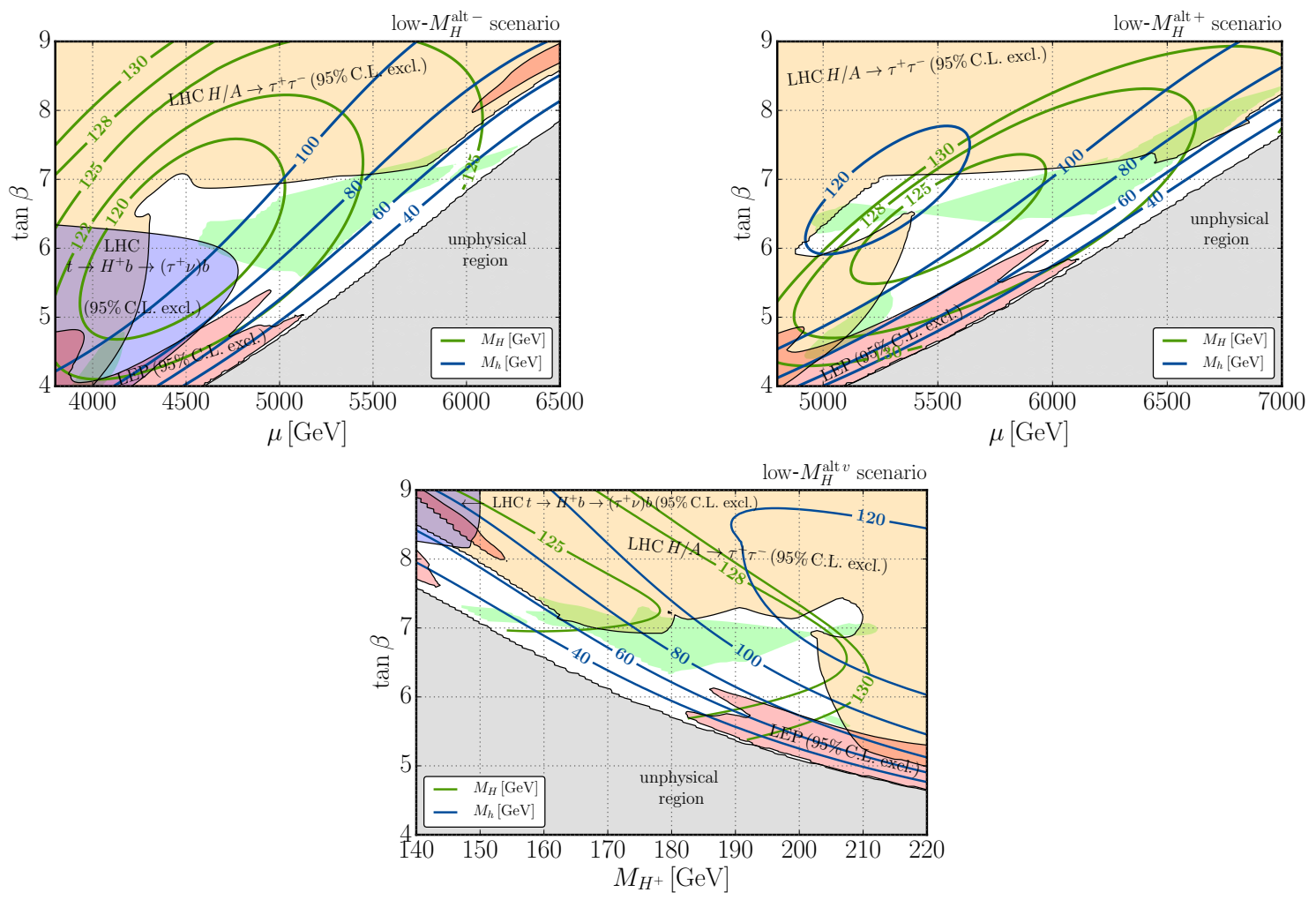

Figure 4: The low- $M_{H}^{\text {alt }-}$ and low- $M_{H}^{\text {alt }+}$ benchmark scenarios in the $\mu$-tan $\beta$ plane with $M_{H^{ \pm}}=155 \mathrm{GeV}$ (upper left), and with $M_{H^{ \pm}}=185 \mathrm{GeV}$ (lower right), and the low- $M_{H}^{\text {altv }}$ benchmark scenario in $\operatorname{the} M_{H^{ \pm}}-\tan \beta$ plane with $\mu=6000 \mathrm{GeV}$ in the lower row. For the color coding and line styles see text.

While being "squeezed" from different searches, Fig. 4 shows that the heavy Higgs case remains a valid option with the interesting feature of a light $\mathscr{C} \mathscr{P}$-even Higgs below $125 \mathrm{GeV}$. We hope that the new benchmark scenarios facilitate the search for these light Higgs bosons as well as for the heavier, not yet discovered Higgs bosons in Run II. 


\section{Conclusinos}

We have briefly reviewed the case that the Higgs boson observed at $\sim 125 \mathrm{GeV}$ is the heavy $\mathscr{C} \mathscr{P}$-even Higgs boson in the MSSM, as recently analyzed in Ref. [6]. The analysis uses an eightdimensional MSSM parameter scan to find the regions in the parameter space that fit best the experimental data. It was found that the rates of the heavy $\mathscr{C} \mathscr{P}$-Higgs boson are close to the SM rates, but can still differ by $20 \%$ or more to yield a good fit. Parameters such as $M_{A}, \tan \beta$ or $m_{\tilde{t}_{1}}$ are confined to relatively small intervals, making clear predictions for Higgs and SUSY searches. The light $\mathscr{C} \mathscr{P}$-even Higgs boson escaped the LEP searches via a tiny coupling to SM gauge bosons, and the decay $H \rightarrow h h$ is sufficiently suppressed not to impact too strongly the heavy Higgs boson rates. Three new benchmark scenarios have been reviewed that have been defined to facilitate the experimental searches at the LHC Run II.

\section{Acknowledgements}

I thank P. Bechtle, H. Haber, O. Stål, T. Stefaniak, G. Weiglein and L. Zeune, with whom the results shown here have been derived. I furthermore thank S. Paßehr for helpful discussions. I thank the organizers of $\mathrm{CH}$ arged 2016 for the invitation and the, as always, pleasant and productive atmosphere, as well as for financial support. The work of S.H. is supported in part by CICYT (grant FPA 2013-40715-P) and by the Spanish MICINN's Consolider-Ingenio 2010 Program under grant MultiDark CSD2009-00064.

\section{References}

[1] G. Aad et al. [ATLAS Collaboration], Phys. Lett. B 716 (2012) 1 [arXiv:1207.7214 [hep-ex]].

[2] S. Chatrchyan et al. [CMS Collaboration], Phys. Lett. B 716 (2012) 30 [arXiv:1207.7235 [hep-ex]].

[3] G. Aad et al. [ATLAS and CMS Collaborations], Phys. Rev. Lett. 114 (2015) 191803 [arXiv:1503.07589 [hep-ex]].

[4] H. Nilles, Phys. Rept. 110 (1984) 1; H. Haber, G. Kane, Phys. Rept. 117 (1985) 75; R. Barbieri, Riv. Nuovo Cim. 11 (1988) 1.

[5] S. Heinemeyer, O. Stål and G. Weiglein, Phys. Lett. B 710 (2012) 201 [arXiv:1112.3026 [hep-ph]].

[6] P. Bechtle et al. to appear in Eur. Phys. J. C, arXiv:1608.00638 [hep-ph].

[7] K. Hagiwara, J. Lee and J. Nakamura, JHEP 1210 (2012) 002 [arXiv:1207.0802 [hep-ph]].

[8] R. Benbrik et al. Eur. Phys. J. C 72 (2012) 2171 [arXiv:1207.1096 [hep-ph]].

[9] M. Drees, Phys. Rev. D 86 (2012) 115018 [arXiv:1210.6507 [hep-ph]].

[10] T. Han, T. Li, S. Su and L. Wang, JHEP 1311 (2013) 053 [arXiv:1306.3229 [hep-ph]].

[11] P. Bechtle et al. Eur. Phys. J. C 73 (2013) no.4, 2354 [arXiv:1211.1955 [hep-ph]].

[12] A. Djouadi, Phys.Rept. 459 (2008) 1 [arXiv:hep-ph/0503173].

[13] S. Heinemeyer, Int. J. Mod. Phys. A 21 (2006) 2659 [arXiv:hep-ph/0407244].

[14] S. Heinemeyer, W. Hollik and G. Weiglein, Phys. Rept. 425 (2006) 265 [arXiv:hep-ph/0412214].

[15] J. Gunion and H. Haber, Phys. Rev. D 67 (2003) 075019 [arXiv:hep-ph/0207010]. 
[16] N. Craig, J. Galloway and S. Thomas, arXiv:1305.2424 [hep-ph].

[17] M. Carena, I. Low, N. Shah and C. Wagner, JHEP 1404 (2014) 015 [arXiv:1310.2248 [hep-ph]].

[18] H.Haber, arXiv:1401.0152 [hep-ph].

[19] M. Carena et al., Phys. Rev. D 91 (2015) no.3, 035003 [arXiv:1410.4969 [hep-ph]].

[20] P. Bhupal Dev and A. Pilaftsis, JHEP 1412 (2014) 024 Erratum: [JHEP 1511 (2015) 147] [arXiv:1408.3405 [hep-ph]].

[21] J. Bernon et al., Phys. Rev. D 92 (2015) no.7, 075004 [arXiv:1507.00933 [hep-ph]].

[22] J. Bernon et al., Phys. Rev. D 93 (2016) no.3, 035027 [arXiv:1511.03682 [hep-ph]].

[23] M. Carena et al., Eur. Phys. J. C 73 (2013) no.9, 2552 [arXiv:1302.7033 [hep-ph]].

[24] See: http://www.feynhiggs.de.

[25] S. Heinemeyer, W. Hollik and G. Weiglein, Comput. Phys. Commun. 124 (2000) 76 [arXiv:hep-ph/9812320]; S. Heinemeyer, W. Hollik and G. Weiglein, Eur. Phys. J. C 9 (1999) 343 [arXiv:hep-ph/9812472]; T. Hahn et al., Phys. Rev. Lett. 112 (2014) 14, 141801 [arXiv:1312.4937 [hep-ph]]. H. Bahl and W. Hollik, Eur. Phys. J. C 76 (2016) no.9, 499 [arXiv:1608.01880 [hep-ph]].

[26] G. Degrassi et al. Eur. Phys. J. C 28 (2003) 133 [arXiv:hep-ph/0212020];

[27] M. Frank et al., JHEP 0702 (2007) 047 [arXiv:hep-ph/0611326];

[28] P. Bechtle et al., Eur. Phys. J. C 74 (2014) no.2, 2711 [arXiv:1305.1933 [hep-ph]]; JHEP 1411 (2014) 039 [arXiv:1403.1582 [hep-ph]].

[29] P. Bechtle et al., Comput. Phys. Commun. 181 (2010) 138 [arXiv:0811.4169 [hep-ph]]; Comput. Phys. Commun. 182 (2011) 2605 [arXiv:1102.1898 [hep-ph]]; P. Bechtle et al., Eur. Phys. J. C 74 (2014) no.3, 2693 [arXiv:1311.0055 [hep-ph]]; P. Bechtle et al., Eur. Phys. J. C 75 (2015) no.9, 421 [arXiv:1507.06706 [hep-ph]].

[30] M. Drees et al., Comput. Phys. Commun. 187 (2015) 227 [arXiv:1312.2591 [hep-ph]].

[31] F. Mahmoudi, Comput. Phys. Commun. 178 (2008) 745 [arXiv:0710.2067 [hep-ph]]; Comput. Phys. Commun. 180 (2009) 1718; Comput. Phys. Commun. 180 (2009) 1579 [arXiv:0808.3144 [hep-ph]].

[32] S. Heinemeyer, W. Hollik, G. Weiglein and L. Zeune, JHEP 1312 (2013) 084 [arXiv:1311.1663 [hep-ph]].

[33] R. Barate et al. [ALEPH and DELPHI and L3 and OPAL Collaborations and LEP Working Group for Higgs boson searches], Phys. Lett. B 565 (2003) 61 [arXiv:hep-ex/0306033].

[34] S. Schael et al. [ALEPH and DELPHI and L3 and OPAL Collaborations and LEP Working Group for Higgs Boson Searches], Eur. Phys. J. C 47 (2006) 547 [arXiv:hep-ex/0602042].

[35] V. Khachatryan et al. [CMS Collaboration], JHEP 1410 (2014) 160 [arXiv:1408.3316 [hep-ex]].

[36] CMS Collaboration, CMS-PAS-HIG-14-029.

[37] G. Aad et al. [ATLAS Collaboration], JHEP 1503 (2015) 088 [arXiv:1412.6663 [hep-ex]].

[38] V. Khachatryan et al. [CMS Collaboration], JHEP 1511 (2015) 018 [arXiv:1508.07774 [hep-ex]]. 